\title{
CHAOS SYNCHRONIZATION IN OPTICALLY PROGRAMMABLE
}

\author{
LOGIC CELLS
}

\author{
A. González-Marcos and J.A. Martín-Pereda \\ ETS Ingenieros de Telecomunicación \\ Universidad Politécnica de Madrid. Ciudad Universitaria \\ 28040 Madrid. Spain
}

\begin{abstract}
A possible approach to the synchronization of chaotic circuits is reported. It is based on an Optically Programmable Logic Cell and the signals are fully digital. A method to study the characteristics of the obtained chaos is reported as well as a new technique to compare the obtained chaos from an emitter and a receiver. This technique allows the synchronization of chaotic signals. The signals received at the receiver, composed by the addition of information and chaotic signals, are compared with the chaos generated there and a pure information signal can be detected. Its application to cryptography in Optical Communications comes directly from these properties. The model here presented is based on a computer simulation.
\end{abstract}

Keywords: Chaos. Chaos synchronization. Digital chaos.

\section{INTRODUCTION}

A considerable interest has appeared in the last years concerning the application of chaotic circuits in order to obtain secure communications. The broadband nature of chaotic signals makes them tempting for use in this area and in spread-spectrum situations. The fact that such signals emanate from a deterministic dynamic system lends to the hope that one will also be able to control them sufficiently for many uses. Taken as isolated systems, chaotic dynamics appears to offer many impediments to anyone attempting to put them to use. The main problem to be addressed is then how to synchronize chaotic circuits. It is well known that two identical circuits are able to offer similar chaotic outputs. But if these circuits are not synchronized their output signals are not valid to be employed in a communication system. The main reason is the strong dependence of the obtained chaos on the initial and boundary conditions of the chaos generator circuit. Two chaotic signals with the same characteristics may have different values at any particular time if they are generated independently. Some additional conditions have to be imposed to the system in order to obtain identical chaotic signals at any time. One of these conditions is the synchronization.

Several attempts have been made in this direction. The idea that chaotic systems could synchronize was first put forth in a paper almost ten years ago ${ }^{1}$. Several authors have followed the lines indicated in that paper. Pecora and Carroll ${ }^{2-4}$ demonstrated the possibility of sinchronizing chaotic subsystems with a common driving signal. Their idea was to decompose the chaotic dynamical system in two subsystems, "driving" and response" subsystems. The driving subsystem is composed by two state variable components whereas the second one just has one and uses as input signal one of the state components of the first subsystem. Several authors have followed this idea and schemes using Chua's circuits are reported in the literature 5 .

Another additional point needs to be considered. It concerns the characteristics of the chaotic signal to be employed. Almost in any of the reported situations the obtained chaos is analog. Although all physical systems are really analog, communication and computer systems are nowadays digital. The way to use analog signals in digital systems in to make the conversion analog/digital. This idea has been employed in any case where an application to communication is needed. It should be useful to obtain digital chaos from the very beginning of the process and to employ it with the same requirements of the information signals.

The purpose of this paper is to present a way to obtain digital chaos and how to synchronize two chaotic systems. The main scheme of the proposed system is shown in Fig. 1. Two identical chaos generators are located at emitter and receiver. Information signal is added at the receiver and transmitted to the network. This composed signal is detected at the receiver and processed with another chaotic signal obtained there. The resulting signal is the information generated at the emitter. This configuration is the basis of our system:

\section{OPTICAL PROGRAMMABLE LOGIC CELL (OPLC)}

The main block of our chaos generator is an Optically Programmable Logic Cell employed previously by us as a part of a possible optical computer ${ }^{6-10}$. Although this structure has been reported in several places, some of its principal 
characteristics will be here presented again. Its main characteristic is the logic processing of two input binary signals, governed by two control signals. Two outputs give logical functions of these inputs. The type of processing is related to the

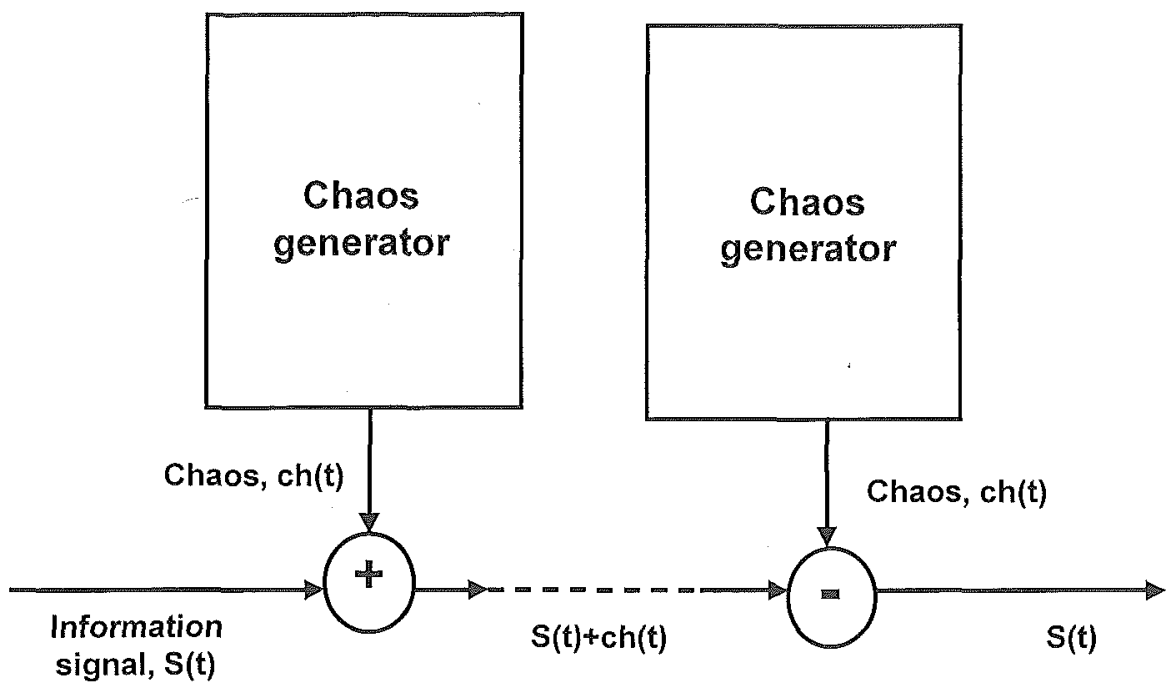

Fig.1.- Proposed security communication system.

eight main Boolean Functions, namely, AND, OR, XOR, NAND, NOR, XNOR, ON and OFF. The programmable ability of the two outputs, as it has been described, allows the generation of several data coding for optical transmission. Moreover, as it was shown, this circuit has the possibility to the generation of periodic and even chaotic solutions. A precise analysis of the output characteristic versus the main variable parameters, as control signal level and data signal level, has been reported [6].

With this configuration, the above mentioned digital character of the signal is directly obtained. Its main blocks are shown in Fig. 2. Two devices with a non-linear behaviour, $\mathrm{P}$ and $\mathrm{Q}$, compose the circuit. The outputs of each one of them correspond to the two final outputs, $\mathrm{O}_{1}$ and $\mathrm{O}_{2}$, of the cell. Four are the possible inputs to the circuit. Two of them are for input data, $\mathbf{I}_{1} \mathrm{e}_{2}$, and the other two, $\boldsymbol{g}$ and $\boldsymbol{h}$, for control signals. The way these four inputs are arranged inside the circuit is also represented in Figure 2. A practical implementation we have carried out of the processing element has been based on an optoelectronical configuration. Lines in Fig. 2 represent optical multimode fibers. The indicated blocks, placed in order to combine the corresponding signals, are conventional optical couplers. In this way, optical inputs arriving to the individual devices are multilevel signals. The characteristics of the non-linear devices are also shown in Fig. 2. Device Q, corresponds to a thresholding or switching device, and device $P$ is a multistate device, being the response of this non-linear optical device the one represented in Fig. 2. This response is similar to the behaviour of a SEED device.

\section{CHAOS GENERATION FROM AN OPLC}

A non-linear behaviour is expected if some kind of feedback is applied to this cell. The feedback we have applied to the system, among the different possibilities, is the one going from the output $\mathrm{O}_{1}$ of P-device (see Fig. 2 ) to the control input, $g$, of P-device. No other additional control signal has been used. A

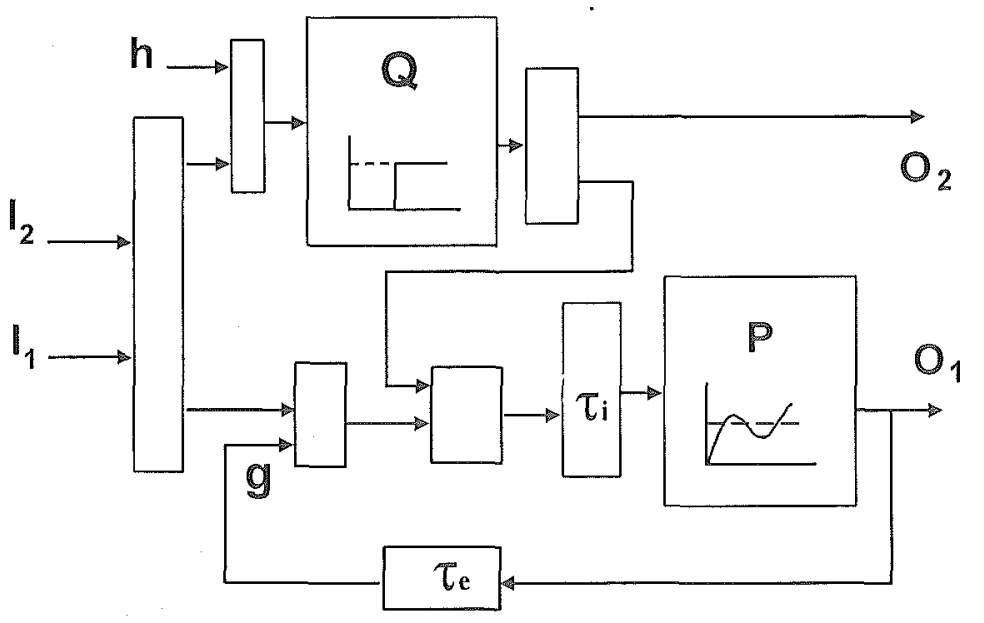

Fig.2.- OPLC main blocks 
chaotic output is obtained when the internal response time is made equal to zero or is much smaller than the external one. Some results have been reported by us ${ }^{11-12}$.

In order to characterize the obtained chaotic signal, conventional methods are difficult to be applied here. The above results constitute a Time Series from where a chaos measure should be obtained. But the correct phase-space representation is not possible to be grasped from these results in a straightforward way. We do not even know what the adequate phase-space variables are and it is not even known how many variables are needed to fully describe the dynamics of this particular system. There is fortunately a partial answer to this problem that has been applied successfully in a large number of experimental investigations. The basic idea is that if the fundamental phase-space variables are $\mathbf{x}$ and $\mathbf{x}^{\prime}$, to study the evolution of the system numerically, $x$ and $x^{\prime}$ have to be follow as functions of $t$. But since $x^{\prime}=[x(t+\Delta t)-x(t)] / \Delta t$ in the limit as $\Delta t \rightarrow 0$, a knowledge of $x(t+\Delta t)$ is equivalent to a knowledge of $x^{\prime}$. In other words, a knowledge of a trajectory of points $[x(t), x(t+\Delta t)]$ is equivalent to a knowledge of the trajectory of points $\left[\mathrm{x}(\mathrm{t}), \mathrm{x}^{\prime}(\mathrm{t})\right]$. As a consequence, a phase-space trajectory

$$
x(t)=\left[x_{1}(t), x_{2}(t), \ldots, x_{n}(t)\right]
$$

is replaced by a trajectory in an artificial phase space with points given by

$$
y(t)=[y(t), y(t+\Delta t), \ldots, y(t+m \Delta t)]
$$

where $y(t)$ is any one of the phase-space variables $x_{i}(t)$. Thus from a set of measurements of a single quantity $y(t)$ we can construct a sequence of points in an artificial phase space

$$
\begin{gathered}
x(t)=[y(t), y(t+\Delta t), \ldots, y(t+m \Delta t] \\
x(t+\Delta t)=[y(t+\Delta t), \ldots, y(t+(m+1) \Delta t]
\end{gathered}
$$

With the data we have, the first problem to solve is how to operate with our digital signal where just two values, " 0 " and "1", are present. If we adopt just this output as possible values for $y$, the resulting plot at the phase space should be concentrated on just four points, namely, $(0,0),(0,1),(1,0)$ and $(1,1)$. Almost no information could be obtained from it. Hence a new technique has to be implemented.

The method we have adopted is to group sets of four consecutive bits and to convert them to their corresponding hexadecimal values. Hence, a sequence of zeroes and ones is converted to a new string of hexadecimal values, namely, $0,1,2$, ...., 15. For example, "0010" would be a "2", "1001" a "9" and "1110" a "14". Four divides the total number of data, but much more information can be obtained from them than with simple binary signals. A diagram, similar to the $t_{i+1}$ versus $t_{i}$ in analogue signals, can be drawn in this way. In the case of periodic signals, a closed configuration is obtained. But in the case of chaotic signals, no definite pattern would be obtained.

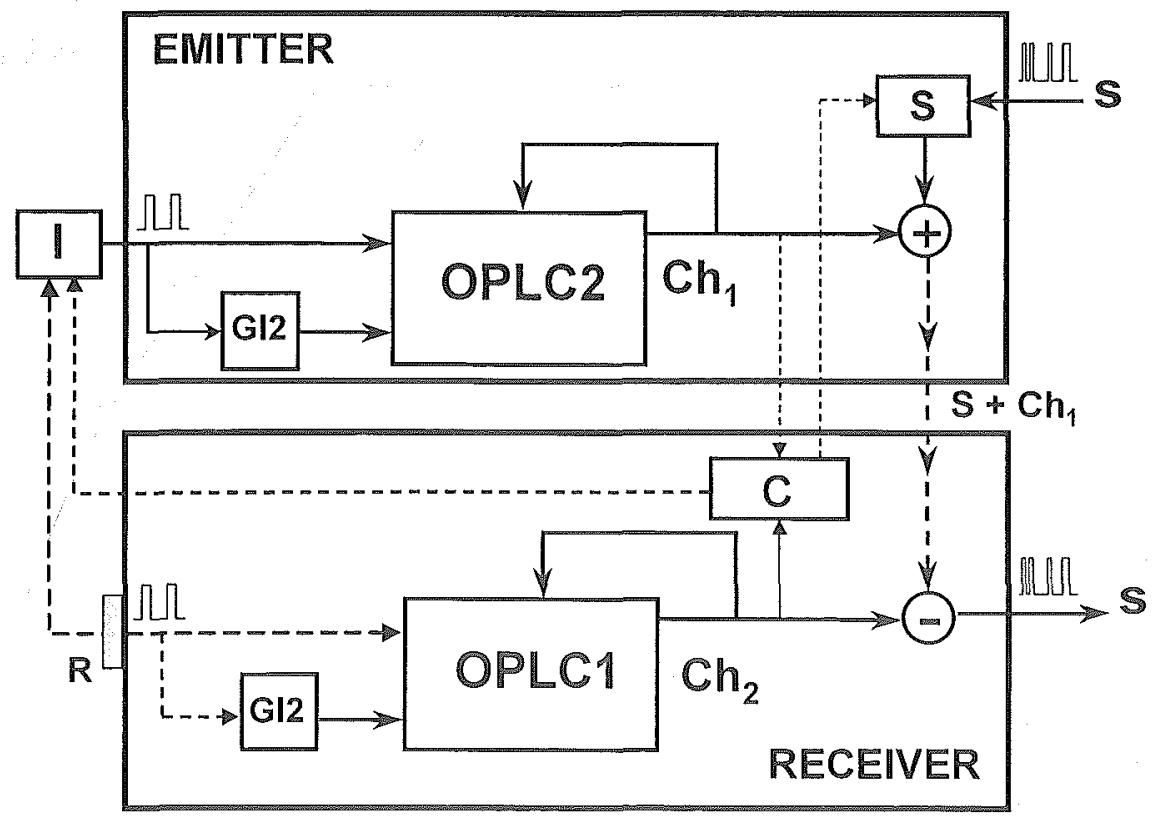

Fig. 3.- Internal configuration of the communication system of fig.1 
A further point needs to be considered. It is the one concerning the justification that the preceding quantity, namely the hexadecimal sequence, represents the same behavior of the system than the previously obtained binary one. But this situation is equivalent to the reverse one: to convert a chaotic analog signal into a digital one. As it is well known from digital communications, any analog signal can be quantized and from this quantization to obtain a digital signal with the same main properties than the initial analog one. In our present case, we have accomplished the opposite operation, namely, to convert a digital signal into an analog quantized one with sixteen possible levels. And, according to digital communication signal processing theory, both representations are equivalent.

\section{SYNCHRONIZATION OF CHAOTIC OPLCs}

If two identical cells with feedback, as the above mentioned, are parallel connected and the same signal arrives to their inputs, an identical chaos is obtained at their outputs. This situation corresponds to two identical and ideal configurations working under identical conditions.

The behaviour becomes critical when the simulation tries to be close to a real situation. In this case, if both systems are not feed by exactly the same signal, the obtained outputs, although chaotic, could be different. Hence, no possible relation between then should be feasible. In a general situation, both systems, emitter and receiver, are located at different places. As a consequence, there is no possibility to introduce exactly the same input signals at the same time to their corresponding input ports. This is because although a common signal generator could send the same train of pulses to both cells, the arriving times to them can be different. The time need to get the first cell is known if this generator is at the receiver place. But the time when the signal arrives to the second cell may not be known. This is the most general case. Several solutions could be implemented to overcome this fact. We have reported a first approach to this problem in ${ }^{12}$. The solution we have adopted in this work is presented in Fig. 3.

The same input driving signal is sent to both cells. A periodic train of pulses of short duration composes this signal and its main objective is to determine the beginning of the control input signal for the OPLCs. If both are exactly the same one output signals from our OPLCs will have the same chaotic characteristics and will be able to be taken for the final signal processing. A new circuit, $\mathrm{C}$ in Fig. 3, will compare both signals. Although there is a time delay between the time the initial signals arrive to both circuits, this time is compensated at the receiver because the same time will need the signal from the emitter to reach the receptor. If the signals processed at $\mathrm{C}$ are different, a new signal is sent to $\mathrm{I}$, generator of the initial pulses. A new train will start again as well as the whole process.

When the received signals at $\mathrm{C}$ are the same, and so both circuits are synchronized, $\mathrm{C}$ sends an information signal to the emitter. At this moment, information signal is added to the chaotic one and both transmitted to the receiver. By a simple subtraction information is extracted.

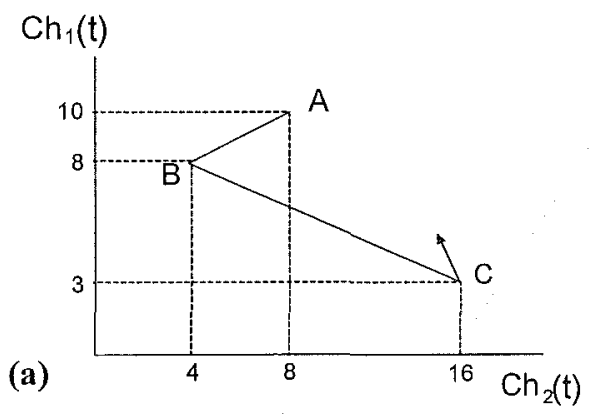

(b)

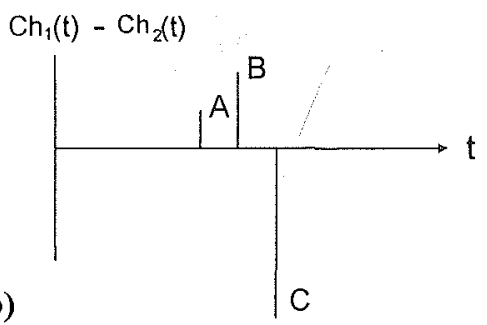

Fig. 4.- Methods used to compare chaos signals. a) From hexadecimal real time data. b) Subtraction of chaos signals .
Two methods have been implemented in order to know if both chaotic signals are the same. The first one is shown in Fig. 4.a. Chaos signal from OPLC1 is represented at the $\mathrm{x}$-axis and the one from OPLC2 at the $y$-axis. A hexadecimal representation, as before, was taken. In each instant time, the value of A particular case is represented in Fig. 5.

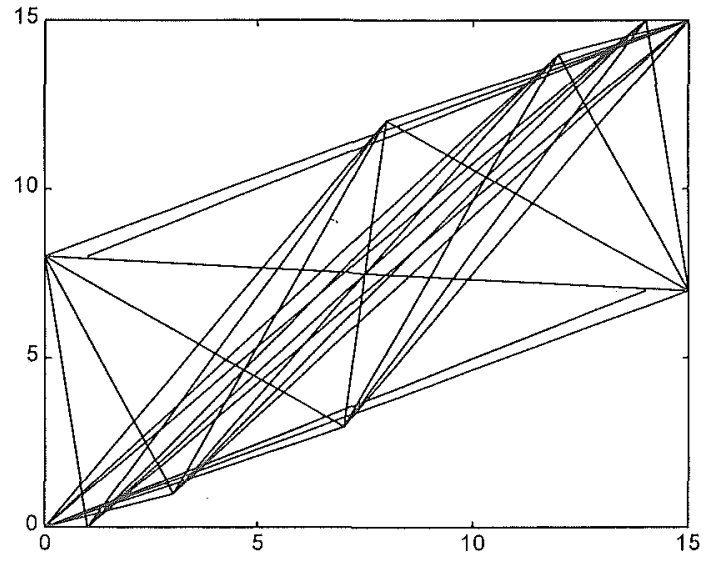

Fig. 5.- Aplication of method of fig. 4.a) 
The second method is just to represent the difference "Chaos from OPLC1 - Chaos from OPLC2" versus time. An example is given at Fig. 4.b. A final result, for a particular case, is shown in Fig. 6. It shows "Chaos from OPLC1 minus chaos from OPLC2" versus time. As it can be seen, after a certain number of steps, synchronization is obtained.

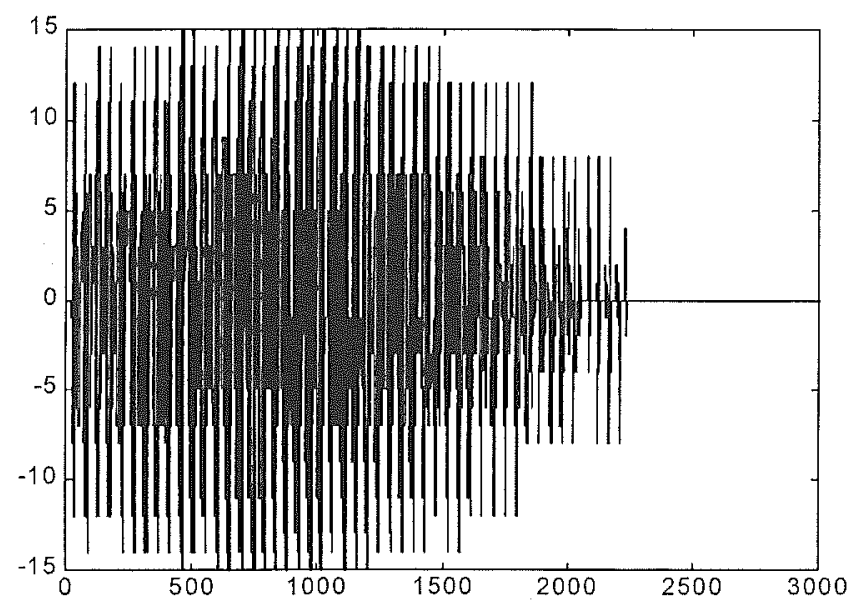

Fig. 6.- Subtraction of chaos signal from emitter and receiver. After a time period it can be seen that syncronizing is achieved.

Moreover, in order to characterise the obtained chaotic signals a Fourier transform was obtained in real time. Signals coming from emitter and receiver are compared at C. Some of the obtained results, corresponding to two different time intervals are shown in Fig. 7.a and 7.b. These signals are an information added to the above mentioned first method.
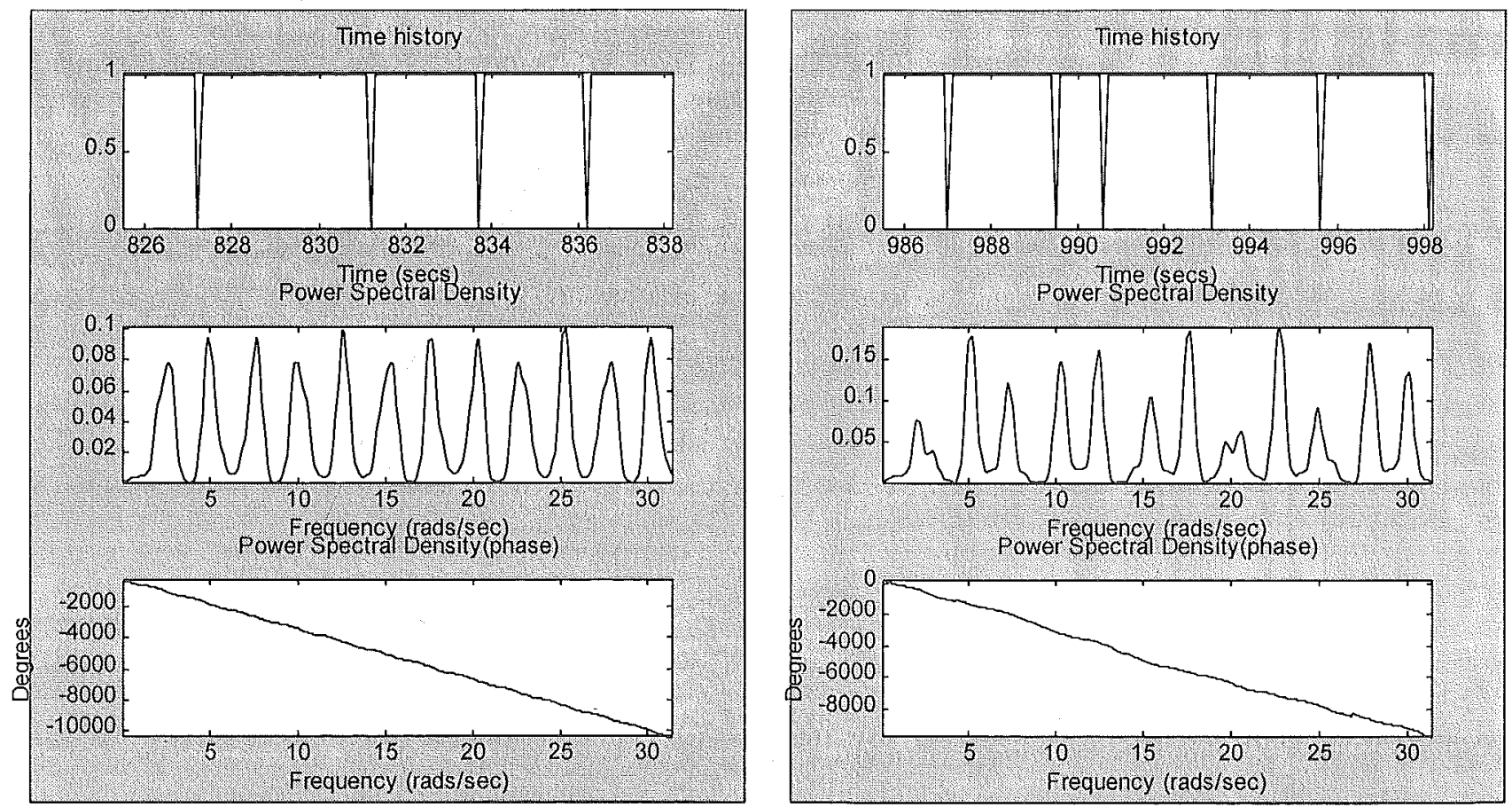

Fig. 7.- Fourier transform at two time intervals of the chaotic signal. 


\section{CONCLUSIONS}

A new method to synchronize chaotic circuits has been reported. The method is based on the use of Optically Programmable Logic Cells as chaos generators. Although these cells have been studied previously, some further studies are needed in order to determine the characteristics of the obtained chaos when the initial driving signal changes. The dependence with its parameters deserves also a deeper study. Moreover, the added delay time has been set to a certain value and if it is not the adequate one, the cell is reset to zero and the process start again. An study of how small and continuos changes affect the results are needed.

\section{ACKNOWLEDGEMENTS}

This work has been partially supported by CAM, grant 07T/0017/1997.

\section{REFERENCES}

1.- V.S. Afraimovich, N.N. Verichev and M.I. Rabinovich, "Stochastic synchronization of oscillations in dissipative systems", Inv. VUZ. Rasiofiz. RPQAEC 29, 795-803, 1986.

2.- L.M. Pecora and T.L. Carroll, "Synchronization in Chaotic Systems", Physical Review Letters 64, 821, 1990.

3.- L.M. Pecora, "Overview of Chaos and Communications Research". in "Chaos in Communications", SPIE Proceedings, 2038, 2-25, SPIE. Bellingham, WA. 1993.

4.- T.L. Carroll and L.M. Pecora, "Synchronizing Chaotic Circuits", IEEE Trans. on Circuits and Systems, 38, 453, 1991.

5.- Several examples are given in "Chua's Circuit: A Paradigm for Chaos". Ed.: R.N. Madan. World Scientific Series on Nonlinear Science. World Scientific. London. 1993.

6. A. González-Marcos and J.A. Martín-Pereda. "Quasi-chaotic digital behavior in an optically processing element". SPIE, 2038, 67-77, 1993.

7. A. González-Marcos \& J.A. Martín-Pereda. "Sequences of bifurcations and transition to Chaos in an OpticallyProcessing Element". In Optical Computing, B.S. Wherrett, Ed., pp.621-624,. Inst. Phys. Conf. Series. No. 139 Part IV. 1995.

8.- J.A. Martín-Pereda \& A. González-Marcos: "Some Connections between Neurophysiology and Optical Computing based on the Theory of Complexity". En "Fluctuation Phenomena: Disorder and Nonlinarity". Eds.: J. Bishop \& L. Vázquez. pp. 107-113. World Scientific Press. Singapur. 1995.

9.- J.A. Martín-Pereda \& A. González-Marcos, "Digital chaos analysis in optical logic structures". SPIE's Photonics East'95 Symposium. Philadelphia, PA. 22-26 October, 1995.

10.- A. González-Marcos \& J.A. Martín-Pereda, "Digital Chaotic Output from an Optically-Processing Element". Optical Engineering., 35 (2), 525-535, 1996.

11.- J.A. Martín-Pereda, A. González-Marcos \& C. Sánchez-Guillén, "Synchronizing Chaotic OpticallyProgrammable Digital Circuits". Globecom 96. IEEE Global Telecommunications Conference, London. November, 1996.

12.- A. González-Marcos \& J.A. Martín-Pereda, "Digital chaos synchronization in optical networks". In "Optical Network Design and Modelling II', eds. G. De Marchis \& R. Sabella. Chapman and Hall. 1998 (in press). 